\title{
The weak INTEGRAL bursts GRB 040223 and GRB 040624 : an emerging population of dark afterglows ${ }^{\star}$
}

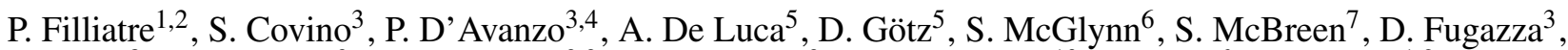 \\ A. Antonelli ${ }^{8}$, S. Campana ${ }^{3}$, G. Chincarini ${ }^{3,9}$, A. Cucchiara ${ }^{3}$, M. Della Valle ${ }^{10}$, S. Foley ${ }^{6}$, P. Goldoni ${ }^{1,2}$, L. Hanlon ${ }^{6}$, \\ G. Israel ${ }^{8}$, B. McBreen ${ }^{6}$, S. Mereghetti ${ }^{5}$, L. Stella ${ }^{8}$, and G. Tagliaferri ${ }^{3}$ \\ ${ }^{1}$ Laboratoire Astroparticule et Cosmologie, UMR 7164, 11 place Marcelin Berthelot, 75231 Paris Cedex 05, France \\ e-mail: filliatr@yahoo.fr \\ 2 Service d'Astrophysique, CEA/DSM/DAPNIA/SAp, CE-Saclay, Orme des Merisiers, Bât. 709, 91191 Gif-sur-Yvette Cedex, France \\ 3 INAF, Osservatorio Astronomico di Brera, via E. Bianchi 46, 23807 Merate (LC), Italy \\ ${ }^{4}$ Universita“ Insubria, Dipartimento di Fisica e Matematica, via Valleggio 11, 22100 Como, Italy \\ 5 INAF - Istituto di Astrofisica Spaziale e Fisica Cosmica di Milano, via E. Bassini 15, 20133 Milano, Italy \\ ${ }^{6}$ School of Physics, University College Dublin, Dublin 4, Ireland \\ 7 Astrophysics Missions Division, Research Scientific Support Department of ESA, ESTEC, Noordwijk, The Netherlands \\ 8 INAF, Osservatorio Astronomico di Roma, via Frascati 33, Monteporzio Catone, 00040 Rome, Italy \\ 9 Università degli Studi di Milano-Bicocca, piazza dell'Ateneo Nuovo 1, 20126 Milano, Italy \\ 10 INAF, Osservatorio Astrofisico di Arcetri, largo E. Fermi 5, 50125 Firenze, Italy
}

Received 19 August 2005 / Accepted 17 November 2005

\section{ABSTRACT}

We report here $\gamma$-ray, X-ray and near-infrared observations of GRB 040223 along with $\gamma$-ray and optical observations of GRB 040624 . GRB 040223 was detected by INTEGRAL close to the Galactic plane and GRB 040624 at high Galactic latitude. Analyses of the prompt emission detected by the IBIS instrument on INTEGRAL are presented for both bursts. The two GRBs have long durations, slow pulses and are weak. The $\gamma$-ray spectra of both bursts are best fit with steep power-laws, implying they are X-ray rich. GRB 040223 is among the weakest and longest of INTEGRAL GRBs. The X-ray afterglow of this burst was detected $10 \mathrm{~h}$ after the prompt event by XMM-Newton. The measured spectral properties are consistent with a column density much higher than that expected from the Galaxy, indicating strong intrinsic absorption. We carried out near-infrared observations $17 \mathrm{~h}$ after the burst with the NTT of ESO, which yielded upper limits. Given the intrinsic absorption, we find that these limits are compatible with a simple extrapolation of the X-ray afterglow properties. For GRB 040624, we carried out optical observations $13 \mathrm{~h}$ after the burst with FORS 1 and 2 at the VLT, and DOLoRes at the TNG, again obtaining upper limits. We compare these limits with the magnitudes of a compilation of promptly observed counterparts of previous GRBs and show that they lie at the very faint end of the distribution. These two bursts are good examples of a population of bursts with dark or faint afterglows that are being unveiled through the increasing usage of large diameter telescopes engaged in comprehensive observational programmes.

Key words. gamma-rays: bursts - gamma-rays: observations

\section{Introduction}

The emergence of comprehensive observational follow-up programmes for GRBs means that it is possible to have, for a particular GRB, a set of multiwavelength observations going from the $\gamma$-ray to the radio domain, conveying a host of information about the nature of these events (Zhang \& Mészáros 2004). Moreover, the GRB afterglows are a promising tool for

\footnotetext{
* Based on observations made by XMM-Newton and with ESO telescopes under programmes Id 72.D-0480 and 073.D-0255 and with the TNG. INTEGRAL is an ESA project with instruments and science data centre funded by ESA member states (especially the PI countries: Denmark, France, Germany, Italy, Switzerland, Spain), Czech Republic, and Poland, and with the participation of Russia and the USA.
}

cosmology, as their absorption spectra lead to the determination of the redshift and the study of the chemical environment of a new set of galaxies (e.g. Fiore et al. 2005), with the possibility of exploration up to the reionisation epoch (Lamb \& Reichart 2000; Cusumano et al. 2005; Tagliaferri et al. 2005b).

However, before they are used for cosmological purposes, it is critical to explore the inhomogeneities among the GRBs, in particular to establish if there are different classes of afterglow behaviour. One such classification distinguishes between bursts with ("bright") and without ("dark") detected optical afterglows.

The estimated fraction of GRBs which did not show any detectable afterglow in the optical band depends strongly on the detection satellite: from less than $10 \%$ for HETE II bursts 
Table 1. Numbers of detected afterglows in the X-ray, optical and radio domains, for a total of 276 GRBs localised before July 2005 (X-ray flashes are excluded).

\begin{tabular}{lrrrrr}
\hline \hline & BeppoSAX & HETE II & INTEGRAL & Swift & Other \\
\hline GRBs & 53 & 55 & 28 & 41 & 99 \\
X-ray & 30 & 10 & 11 & 28 & 10 \\
Optical & 16 & 20 & 9 & 15 & 14 \\
Radio & 10 & 6 & 2 & 5 & 11 \\
\hline
\end{tabular}

(Lamb et al. 2004), to 60\% for events detected by BeppoSAX, XTE or the Interplanetary Network (Lazzati et al. 2002). Popular and non-mutually exclusive explanations are: these bursts have intrinsically faint afterglows in the optical band (e.g. Fynbo et al. 2001a; Lazzati et al. 2002); their decay is very fast (Berger et al. 2002); the optical afterglow is obscured by dust in the vicinity of the GRB or in the star-forming region in which the GRB occurs (e.g. Lamb 2000; Reichart \& Price 2002); their redshift is above 5, so that the Lyman- $\alpha$ absorption by neutral hydrogen in the host galaxy and along the line of sight suppresses the optical radiation of the afterglow (Lamb 2000; Tagliaferri et al. 2005b).

To these physical explanations, one must add the complication that the search techniques may be neither accurate nor quick enough (Lamb et al. 2004). The continuing reduction of observational biases in afterglow searches, and the corresponding increased efficiency of observing facilities, often robotic, devoted to this task has therefore modified the percentage of GRBs with no identified optical or near-infrared (NIR) counterpart. However, the discrepancy between the high-energy (soft $\mathrm{X}$-ray) afterglows and those identified at longer wavelengths (optical and NIR, but also radio) still holds true. In Table 1 we give the numbers of detected afterglows, using the publicly available catalogue maintained by J. Greiner ${ }^{1}$ and Mereghetti \& Götz (2005) for bursts detected by INTEGRAL (Winkler et al. 2003). In order to determine the real percentage of dark afterglows, it is essential to consider in detail some of the various factors affecting the observers' capabilities to single out the counterparts, i.e. the size of the error boxes and the delay in making the position available after the high-energy event. Moreover, for INTEGRAL, one has to add the fact that this satellite preferentially points to the Galactic plane, and therefore tends to select bursts whose afterglow emission is heavily obscured at optical wavelengths.

In this paper, we present and discuss our observations of two GRBs discovered by INTEGRAL, GRB 040223 and GRB 040624. GRB 040223 is located in the direction of the Galactic plane. The X-ray afterglow was detected with $X M M-N e w t o n$, allowing the error box to be reduced significantly. The absorption measured in the X-ray afterglow spectrum is consistent with being about 2.4 times that due to the Galactic plane. However no afterglow was found in our deep observations in the $J, H$ and $K_{\mathrm{s}}$ bands carried out less than $17 \mathrm{~h}$ after the burst. GRB 040624 was very well situated at high Galactic latitude. However, our rather deep observations

\footnotetext{
${ }^{1}$ http://www.mpe.mpg.de/ jcg/grbgen.html
}

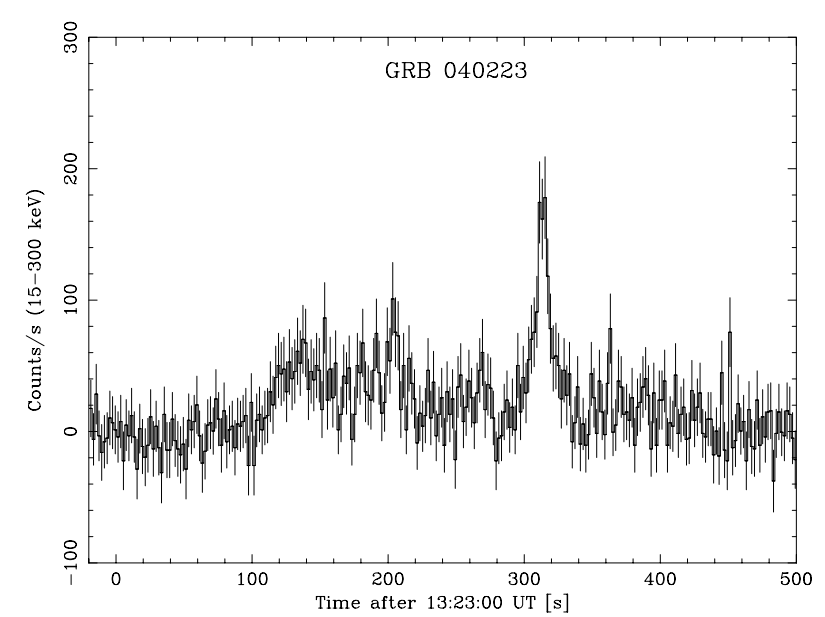

Fig. 1. IBIS/ISGRI light curve of GRB 040223 in the $15-300 \mathrm{keV}$ band in vignetting corrected counts.

carried out within $13 \mathrm{~h}$ of the burst provided only an upper limit on the magnitude of the afterglow in the optical. There are no X-ray observations of the afterglow of GRB 040624. One day after the GRB (Feb. 24.58), radio observations were performed with the Very Large Array (VLA) providing 2- $\sigma$ upper limits of 200 and $174 \mu \mathrm{Jy}$ at 4.9 and $8.5 \mathrm{GHz}$, respectively (Soderberg et al. 2004). We present the $\gamma$-ray characteristics of these two dark bursts in this paper. In addition, we present their afterglow properties and compare them to the afterglow population as a whole.

The errors quoted are at the 1- $\sigma$ level, unless explicitly stated otherwise.

\section{Observations and data analysis}

\subsection{GRB 040223}

\subsection{1. $\gamma$-ray data}

GRB 040223 was detected with IBIS/ISGRI (Imager on Board the INTEGRAL Satellite, Ubertini et al. 2003) at 13:28:10 UTC (Götz et al. 2004), and localised thanks to the INTEGRAL Burst Alert System, IBAS (Mereghetti et al. 2003, 2004a). The IBAS alert was issued at 13:28:30, $30 \mathrm{~s}$ after the beginning of the main peak and $3 \mathrm{~min}$ and $50 \mathrm{~s}$ after the onset of the weak precursor emission. The coordinates are $\alpha=16^{\mathrm{h}} 39^{\mathrm{m}} 31^{\mathrm{s}}$, $\delta=-41^{\circ} 55^{\prime} 47^{\prime \prime}$ (J2000), with an uncertainty of 2!2 (90\% confidence limit, hereafter CL). The Galactic coordinates are $l=$ $341^{\circ} .61, b=3$. 19 .

The light curve $(15-300 \mathrm{keV})$ is given in Fig. 1. The GRB starts at 13:24:40 UT and has a $T_{90}$ duration of $258 \mathrm{~s}$, making it one of the longest GRBs seen by INTEGRAL. It exhibits a multi-peaked structure, with a main peak preceded by two faint precursors.

The peak spectrum (integrated over $1 \mathrm{~s}$ ) and the average spectrum have been extracted for the burst and fit using the latest available response matrices. In the $20-200 \mathrm{keV}$ energy range, the peak flux and fluence are $4 \times 10^{-8} \mathrm{erg} \mathrm{cm}^{-2} \mathrm{~s}^{-1}$ and $2 \times 10^{-6} \mathrm{erg} \mathrm{cm}^{-2}$ respectively. 


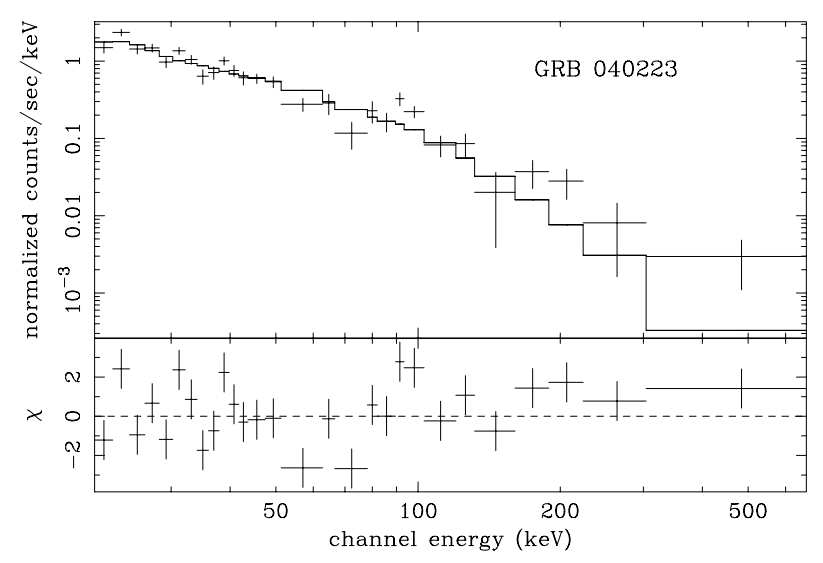

Fig. 2. IBIS/ISGRI average spectrum of GRB 040223 fit with a powerlaw model.

The peak spectrum has a photon index of $1.4 \pm 0.6$, while the photon index for the entire last peak is $1.82 \pm 0.17$. The average spectrum, shown in Fig. 2, can be modelled as a power-law, yielding a photon index of $\Gamma=2.0 \pm 0.2(90 \% \mathrm{CL})$. The results obtained with the germanium spectrometer SPI on INTEGRAL (Vedrenne et al. 2003) are consistent with IBIS (McGlynn et al. 2005).

\subsubsection{The X-ray afterglow}

The INTEGRAL error box was observed by XMM-Newton on February 23, 2004 at 18:21 UTC, slightly less than $5 \mathrm{~h}$ after the burst. This is the fastest response by XMM-Newton to a GRB trigger to date. The observation lasted for $\sim 42 \mathrm{ks}$. We report here on the data collected by the EPIC instrument, consisting of the PN and two MOS cameras (Strüder et al. 2001; Turner et al. 2001).

The afterglow of GRB 040223 (source XMMU J163929.9415601 ) is clearly detected in all cameras (Breitfellner et al. 2004; De Luca et al. 2004). We improved the EPIC astrometry by cross-correlating the serendipitous X-ray sources in the field with stars in the USNO-B1 catalog. The refined coordinates for the X-ray afterglow are $\alpha=16^{\mathrm{h}} 39^{\mathrm{m}} 30^{\mathrm{s}} .17, \delta=-41^{\circ} 55^{\prime} 59^{\prime \prime} .7$ with an error of $1^{\prime \prime} .5$, accounting for the accuracy of the X-ray to optical source superposition, as well as for the positional accuracy of the USNO-B1 catalog. The afterglow faded during the observation, as can be seen in Fig. 3. After checking the consistency of the results from the three detectors, we extracted a combined background-subtracted light curve in the range $1-7 \mathrm{keV}$. The count rate decay is well described $\left(\chi_{v}^{2}=1.05\right.$, 39 d.o.f.) by a power-law $\left(F(t) \propto t^{-\alpha_{\mathrm{X}}}\right)$ with the temporal in$\operatorname{dex} \alpha_{\mathrm{X}}=0.9 \pm 0.1$ (90\% confidence level for a single parameter of interest).

Source and background spectra were extracted. The spectral analysis was performed using XSPEC v11.3. The spectra from the three detectors, shown in Fig. 4, were fit simultaneously. A simple absorbed power-law model yields a good description of the data $\left(\chi_{v}^{2}=0.93,114\right.$ d.o.f. $)$. The best fit values are: $2.60 \pm 0.15(90 \% \mathrm{CL})$ for the photon index, $N_{\mathrm{H}}=(1.60 \pm 0.15) \times 10^{22} \mathrm{~cm}^{-2}$ for the hydrogen column density. This is significantly $(3.7 \sigma)$ higher than the

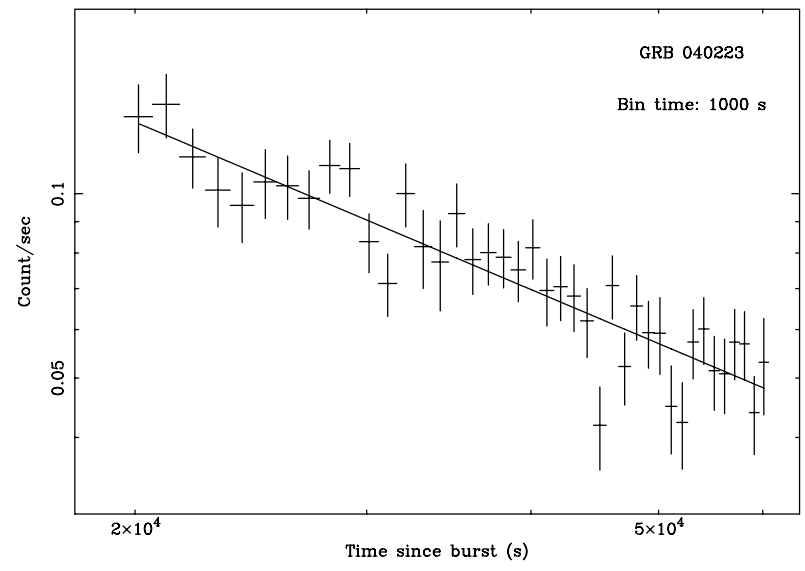

Fig. 3. Background-subtracted light curve of the afterglow of GRB 040223 observed by the EPIC instrument (1-7 keV). The decay is well fit by a power-law $F \propto t^{-\alpha_{\mathrm{X}}}$, with best fit value of $\alpha_{\mathrm{X}}=0.9 \pm 0.1$ $\left(90 \% \mathrm{CL}, \chi_{v}^{2}=1.05,39\right.$ d.o.f.).

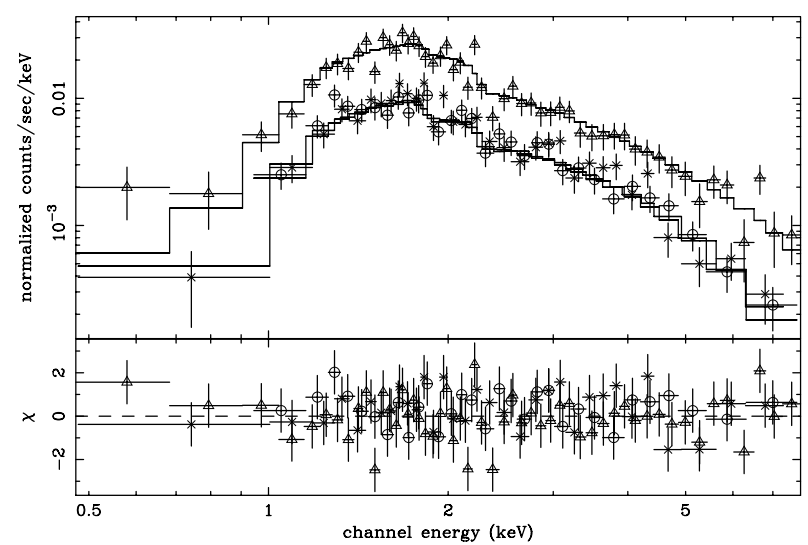

Fig. 4. EPIC spectra of the afterglow of GRB 040223, overlaid with the best fit model convolved through the instrument response matrices and effective area files. Triangles, circles and crosses represent PN, MOS1 and MOS2 data, respectively.

expected contribution of the Galaxy, which is estimated to be $N_{\mathrm{H}}=(6.6 \pm 2) \times 10^{21} \mathrm{~cm}^{-2}$ (Dickey \& Lockman 1990), where the face value and the error are the average and the standard deviation respectively of the 7 nearest available measurements within $1^{\circ}$ of the GRB position. This implies an unabsorbed flux of $\sim 1.0 \times 10^{-12} \mathrm{erg} \mathrm{cm}^{-2} \mathrm{~s}^{-1}$ in the band $0.5-10 \mathrm{keV}$. These results, fully consistent with those found by McGlynn et al. (2005), still hold when two subsets of the data with the same number of counts (having exposure times of $\sim 15 \mathrm{ks}$ and $\sim 27 \mathrm{ks}$, respectively) are considered separately, showing that there is no significant variation in the spectral parameters with time.

We investigated the presence of absorption or emission lines superimposed on the continuum. We added Gaussian lines of fixed width (smaller than the instrumental energy resolution), allowing the central energy in the range $1-5 \mathrm{keV}$ to vary, as well as the line normalisation (in both the emission and absorption cases). We found no significant features. The upper limits $(3-\sigma)$ on the equivalent width of any emission or absorption lines are $\sim 60 \mathrm{eV}$ and $\sim 200 \mathrm{eV}$ in the $1-2.5 \mathrm{keV}$ and $2.5-5 \mathrm{keV}$ ranges, respectively. 
Table 2. Observation $\log$ for GRB 040223. Observing times refer to the middle of the exposures and $\Delta T$ is the time since the GRB. Magnitudes are calibrated following the 2 MASS catalogue. Magnitude limits are at the 3- $\sigma$ level.

\begin{tabular}{llccccc}
\hline \hline $\begin{array}{l}\text { Date } \\
(\mathrm{UT})\end{array}$ & $\begin{array}{l}\Delta T \\
\text { (days) }\end{array}$ & Filter & $\begin{array}{c}\text { Exp } \\
(\mathrm{s})\end{array}$ & $\begin{array}{c}\text { Seeing } \\
\left({ }^{\prime \prime}\right)\end{array}$ & Instrument & Mag \\
\hline 2004 Feb. 24.279 & 0.720 & $J$ & $20 \mathrm{~s}$ & 1.2 & NTT+SofI & $>20.9$ \\
2004 Feb. 24.258 & 0.700 & $H$ & $20 \mathrm{~s}$ & 1.1 & NTT+SofI & $>19.8$ \\
2004 Feb. 24.259 & 0.700 & $K_{\mathrm{s}}$ & $10 \mathrm{~s}$ & 1.1 & NTT+SofI & $>19.9$ \\
2004 Feb. 24.384 & 0.824 & $J$ & $20 \mathrm{~s}$ & 0.687 & NTT+SofI & $>21.1$ \\
2004 Feb. 24.394 & 0.836 & $H$ & $20 \mathrm{~s}$ & 0.67 & NTT+SofI & $>20.4$ \\
2004 Feb. 24.403 & 0.844 & $K_{\mathrm{s}}$ & $10 \mathrm{~s}$ & 0.638 & NTT+SofI & $>20.2$ \\
2004 Feb. 24.538 & 1.821 & $K_{\mathrm{s}}$ & $10 \mathrm{~s}$ & 0.75 & NTT+SofI & $>20.1$ \\
\hline
\end{tabular}

\subsubsection{Optical and near-infrared observations}

The first follow-up observations were carried out by the Faulkes Telescope North $1.57 \mathrm{~h}$ after the GRB in the optical, obtaining a 2- $\sigma$ upper limit of $r^{\prime} \sim 18$ (Gomboc et al. 2004). The GRB was within the Galactic plane, not very far from the Galactic centre, so NIR observations are better suited to overcome the high absorption. The REM robotic telescope (Zerbi et al. 2001; Chincarini et al. 2003) provided NIR upper limits $(2-\sigma)$ of $H=15.5$ and $K=15.016 \mathrm{~h}$ after the GRB trigger (Israel et al. 2004).

A much deeper search for the afterglow in the NIR was carried out with a similar delay using the SofI instrument at the NTT (Tagliaferri et al. 2004; Simoncelli et al. 2004). We made three sets of observations, from 0.7 to 1.8 days after the burst (Table 2). Image reduction was performed following the standard procedures of the ESO - Eclipse package (Devillard 1997). Astrometry and photometric calibration were performed using the 2 MASS catalogue ${ }^{2}$. PSF-photometry of all the objects in the field was carried out using the $I R A F^{3}$ daophot task. A finding chart of the field, with the reported XMM-Newton error box, is shown in Fig. 5.

Within the XMM-Newton error circle of 1'.5 radius we found 6 objects, for which magnitude measurements were carried out in the $K_{\mathrm{s}}$ band at $\Delta T=0.70,0.83$ and 1.8 days. For each of them, we fit a power-law decay over the three epochs of observation in the $K_{\mathrm{s}}$ band and found that a temporal index consistent with zero is always preferred, indicating no source variability. Fixing $\alpha=\alpha_{\mathrm{X}}=0.9 \pm 0.1$ leads to unacceptable $\chi^{2}$ values. Therefore, the limiting magnitudes reported in Table 2 are our 3- $\sigma$ upper limits on the magnitude of the afterglow.

These magnitude limits are not corrected for Galactic absorption. The afterglow lies towards the Galactic plane, rather close to the direction of the Galactic centre. The extinction is high, rather patchy, and uncertain close to the Galactic centre. Using the previously quoted value of $N_{\mathrm{H}}=(6.6 \pm 2) \times 10^{21} \mathrm{~cm}^{-2}$, and the fit of Predehl \& Schmitt (1995), we derive $A_{\mathrm{V}}=3.9$; and hence, using the extinction law of Cardelli et al. (1989) with $R_{\mathrm{V}}=3.1$, we get $E(B-V)_{N_{\mathrm{H}}}=1.2$. The use of the FIRAS

\footnotetext{
${ }^{2}$ http://irsa.ipac.caltech.edu/

${ }^{3} I R A F$ is distributed by the National Optical Astronomy Observatories, which are operated by the Association of Universities for Research in Astronomy, Inc. under cooperative agreement with the National Science Foundation.
}

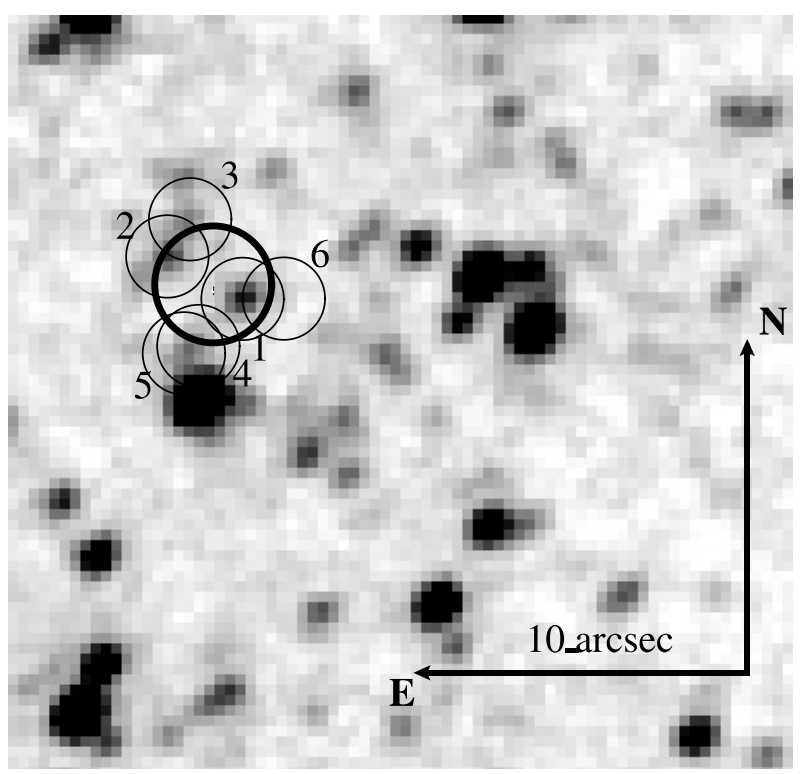

Fig. 5. Finding chart for GRB 040223, with the XMM-Newton error circle shown. Six objects are singled out within the $1-\sigma$ error circle, but none of these show the typical afterglow variability.

dust emission maps at $100 \mu \mathrm{m}$ obtained by Schlegel et al. (1998) leads to a substantially higher value of $E(B-V)_{\mathrm{FIR}}=$ 1.8. For this overestimate of the reddening, typical for low Galactic latitudes, Dutra et al. (2003) proposed a linear correction. In our case, we then get $E(B-V)=1.2$, in full agreement with the value derived from the hydrogen column density. We then derived $A_{R}=3.2, A_{J}=1.1, A_{H}=0.7$ and $A_{K}=0.4$ for the Galactic absorption, and we will adopt these values. It should be noted that the value of $N_{\mathrm{H}}$ derived from the spectrum of the X-ray afterglow is much higher than the one derived for the Galaxy. This is discussed in Sect. 3.

\subsection{GRB 040624}

\subsection{1. $\gamma$-ray data}

GRB 040624 was detected on June 24 at 08:21:35 UT (June 24.348) with IBIS/ISGRI in the $15-200 \mathrm{keV}$ band (Mereghetti et al. 2004b). It was localised with IBAS at $\alpha=13^{\mathrm{h}} 00^{\mathrm{m}} 09^{\mathrm{s}} .9, \delta=-03^{\circ} 35^{\prime} 14^{\prime \prime}$ with an uncertainty of $3^{\prime}(90 \% \mathrm{CL})$, at $59.2^{\circ}$ from the Galactic plane. Data from SPI 
Table 3. Observation log for GRB 040624. Observing dates are referred to the middle of the exposures. Magnitude limits are at the 3- $\sigma$ level.

\begin{tabular}{llccclc}
\hline \hline $\begin{array}{l}\text { Date } \\
(\mathrm{UT})\end{array}$ & $\begin{array}{l}\Delta T \\
(\text { days })\end{array}$ & Filter & $\begin{array}{c}\text { Exp } \\
(\mathrm{s})\end{array}$ & $\begin{array}{c}\text { Seeing } \\
\left({ }^{\prime \prime}\right)\end{array}$ & Instrument & Mag \\
\hline 2004 Jun. 24.906 & 0.558 & $R$ & $2 \times 120$ & 1.2 & TNG+DOLoRes & $>23.8$ \\
2004 Jun. 24.989 & 0.641 & $R$ & $5 \times 30$ & 0.25 & VLT+FORS 2 & $>22.5$ \\
2004 Jun. 25.125 & 0.776 & $R$ & $5 \times 30$ & 0.33 & VLT+FORS 2 & $>24.5$ \\
2004 Jun. 27.987 & 3.638 & $R$ & $5 \times 30$ & 1.0 & VLT+FORS 1 & $>24.3$ \\
2004 Jun. 28.889 & 4.541 & $R$ & $4 \times 120$ & 2.0 & TNG+DOLoRes & $>22.9$ \\
2004 Jul. 07.422 & 13.074 & $R$ & $3 \times 180$ & 1.4 & TNG+DOLoRes & $>24.0$ \\
2004 Jul. 14.919 & 20.571 & $R$ & $2 \times 180$ & 1.3 & TNG+DOLoRes & $>23.4$ \\
\hline
\end{tabular}

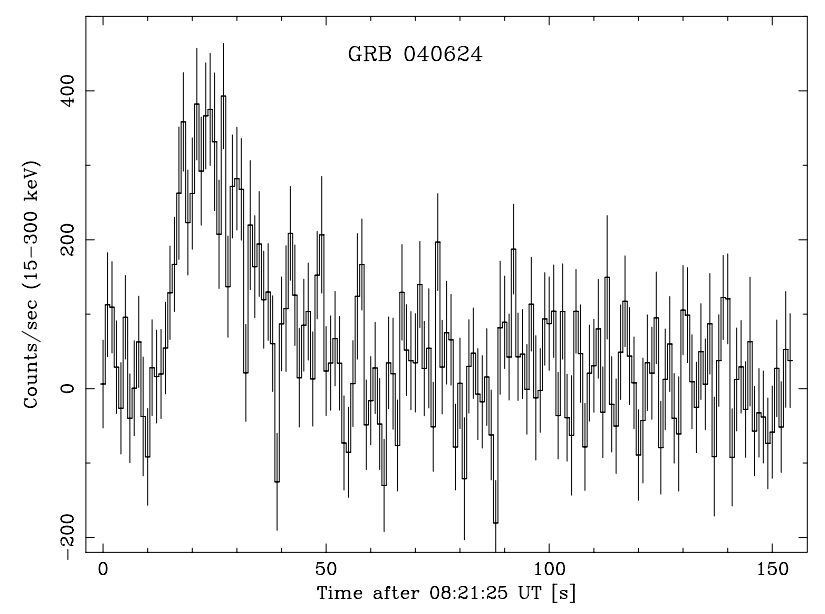

Fig. 6. Light curve of GRB 040624 in the $15-300 \mathrm{keV}$ band.

are unavailable for this burst because the instrument was being annealed.

The IBIS data were analysed using standard INTEGRAL software in a manner similar to GRB 040223 (Sect. 2.1.1). The light curve in the energy range $15-300 \mathrm{keV}$ is given in Fig. 6 and shows the burst consists of one long, slow pulse. The duration is $T_{90}=46 \mathrm{~s}$.

The spectrum obtained for GRB 040624 is shown in Fig. 7. Each bin contains a minimum of 20 counts per bin. The peak flux (integrated over one second) in the energy range $20-200 \mathrm{keV}$ is $4 \times 10^{-8} \mathrm{erg} \mathrm{cm}^{-2} \mathrm{~s}^{-1}$. The fluence in the same energy range is $1.0 \times 10^{-6} \mathrm{erg} \mathrm{cm}^{-2}$. The spectrum is best fit by a power-law with photon index $\Gamma=2.1 \pm 0.2$.

\subsubsection{Optical observations}

The first follow-up observations were carried out with the the $152 \mathrm{~cm}$ telescope of Bologna University, yielding a magnitude limit of $R_{\mathrm{c}}=20.5$ at $11.8 \mathrm{~h}$ (Piccioni et al. 2004), and by the $1.5 \mathrm{~m}$ Observatorio de Sierra Nevada (OSN) telescope, yielding a magnitude limit of $R \sim 21$ at $12.5 \mathrm{~h}$ (Gorosabel et al. 2004). The GRB was located far from the Galactic plane at $l=307^{\circ} 19, b=59^{\circ} .21$, where the optical extinction is negligible. We estimate $A_{\mathrm{V}}=0.8$ and $A_{R}=0.06$ (see Sect. 2.1.3). Our group followed the field in the optical domain with DOLoRes at TNG, and FORS $1 / 2$ at the VLT as reported in Table 3, with time delays ranging from 0.553 to 20.571 days (Fugazza et al. 2004; D’Avanzo et al. 2004). The late time

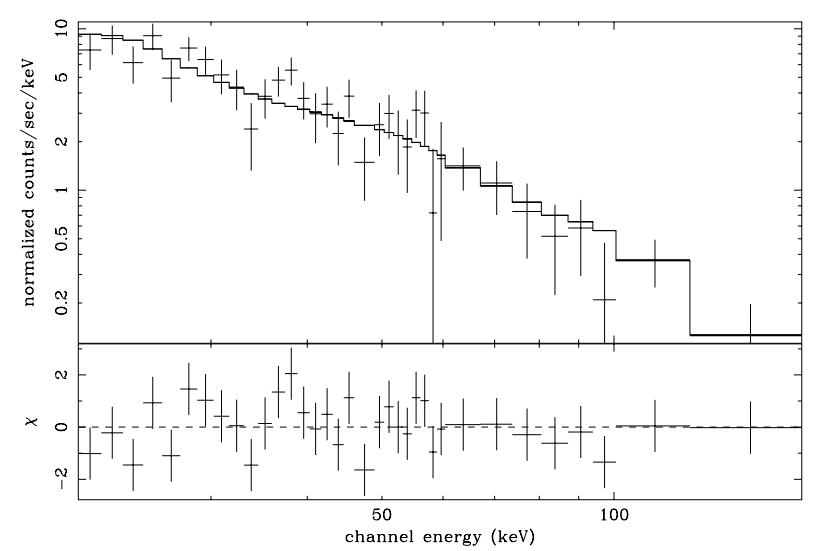

Fig. 7. IBIS/ISGRI average spectrum of GRB 040624 fit by a powerlaw model.

observations had the possibility of detecting an underlying supernova.

\section{Discussion}

\subsection{The prompt event}

The two bursts, GRB 040223 and GRB 060624, are rather faint and about $2 / 3$ of INTEGRAL GRBs have larger peak fluxes (Mereghetti \& Götz 2005). The steep power-law spectra (of $\Gamma=$ $2.0 \pm 0.2$ for GRB 040223 and $\Gamma=2.1 \pm 0.2$ for GRB 040624) indicate that they are most likely X-ray rich GRBs (Barraud et al. 2003). Indeed, extrapolating this steep power-law spectrum, we get the flux ratio $S_{7-30 \mathrm{keV}} / S_{30-400 \mathrm{keV}} \sim 0.5$ for GRB 040223 , while for classical GRBs this value is smaller than 0.3 .

Using a large sample of bursts, McBreen et al. (2002b) showed that the cumulative light curves of GRBs are approximately linear over the main emission period of the burst, indicating an almost constant energy output by the central engine. The normalised cumulative profiles of GRB 040223 and GRB 040624 for the main emission periods, presented in Fig. 8, exhibit similar profiles showing that most of the emission evolves linearly with time.

The temporal properties of the pulses in GRBs have been determined and a relationship found between the pulse properties, the number of pulses and $T_{90}$ (Quilligan et al. 2002; McBreen et al. 2002a). The main pulse of both GRB 040223 and GRB 040624 have slow rise and fall times, and their pulse properties, along with their $T_{90}$ durations are quite consistent 


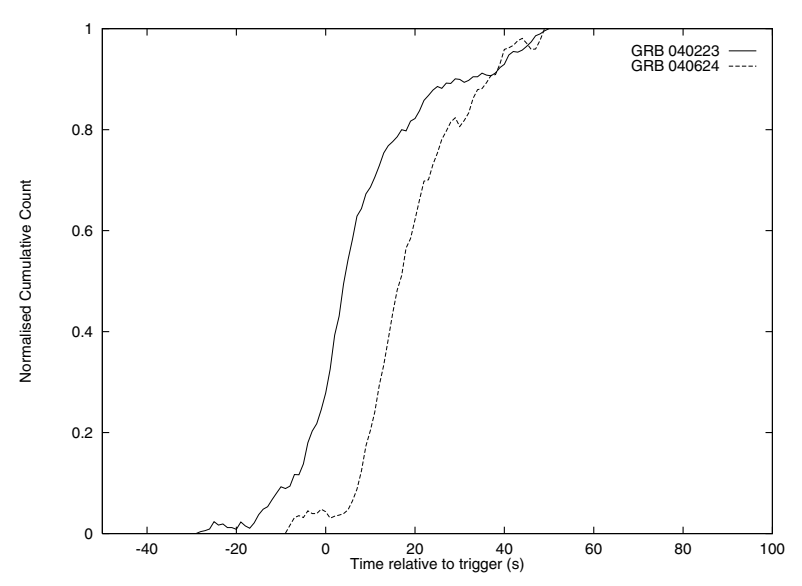

Fig. 8. Normalised cumulative light curves of GRB 040223 (solid line) and GRB 040624 (dashed line).

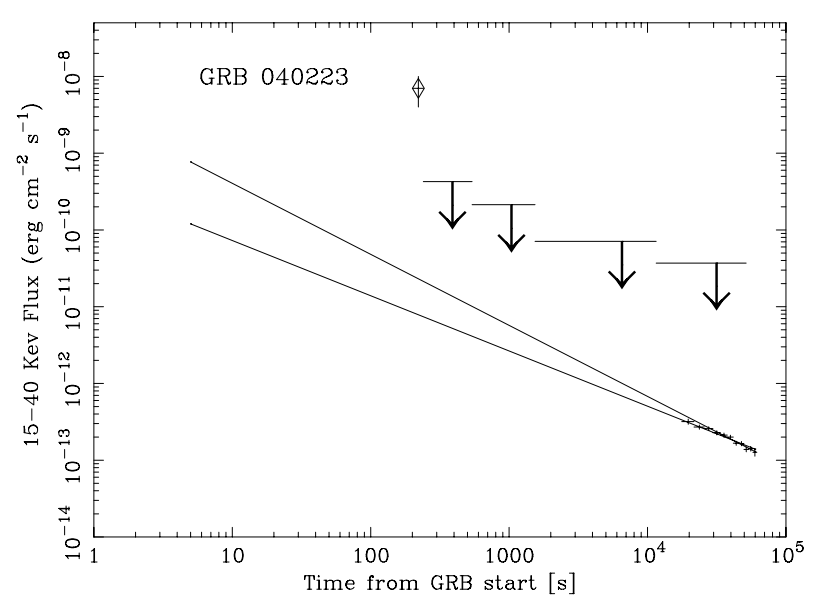

Fig. 9. Prompt emission of GRB 040223 measured by INTEGRAL (diamond) compared with the extrapolation to earlier times of the X-ray afterglow emission detected by XMM-Newton within the limits of the measurements (two solid lines). The arrows indicate IBIS/ISGRI 5- $\sigma$ upper limits for the emission in the direction of the GRB.

with the pulse timing diagrams obtained by McBreen et al. (2002a). GRB 040106 is another example of an INTEGRAL burst that fits on the timing diagram (Moran et al. 2005).

In many cases the location of a GRB discovered with IBAS can be monitored for a few hours to days after and before the burst, thanks to the large FOV of INTEGRAL and its typically long observation time. For GRB 040223, we have performed a search for precursors and early $\gamma$-ray afterglow in IBIS/ISGRI data using the Off-line Scientific Analysis (OSA). We have not been able to detect any precursors or early high-energy afterglow emission. This burst is, however, much weaker than those for which high energy afterglows have been reported (GRB 920723: Burenin et al. 1999; GRB 940217: Hurley et al. 1994; GRB 980923: Giblin et al. 1999).

Following the work of Frontera et al. (2000) on BeppoSAX data, we have compared the X-ray afterglow light curve with the prompt $\gamma$-ray emission of GRB 040223 in order to evaluate the relationship between them. Frontera et al. (2000) report that the X-ray afterglow starts at about $50 \%$ of the GRB duration and its fluence, as computed from the WFC light curve, is consistent with the decay law found from the afterglow NFI observations. In our case the extrapolation of the X-ray afterglow is well below the $\gamma$-ray flux and extrapolates to a time earlier than the GRB. We should point out that Tagliaferri et al. (2005a, see also Chincarini et al. 2005; Nousek et al. 2005) have shed new light on the relationship between the GRB and the early X-ray afterglow using Swift-XRT data. In particular the X-ray afterglows of some GRBs in their sample are characterised by a rapid fall-off ( $\alpha_{X} \geqq 2.5$ ) in the first few hundred seconds, followed by a less rapid decline $\left(\alpha_{\mathrm{X}} \sim 1\right)$ lasting several hours. The behaviour of GRB 040223 is compatible with this picture and not with that derived from the BeppoSAX GRBs.

\subsection{The optical/NIR afterglow}

We compared our magnitude limits for GRB 040223 and GRB 040624 with the magnitudes of observed afterglows that are listed in Table 4 selected according to the following criteria:

- a photometric measurement of the optical counterpart was made in the first $30 \mathrm{~h}$ in good observing conditions. This timing constraint had two motivations: first, to avoid a bias towards bright afterglows that late observations tend to select (as faint afterglows are then more likely to be below the detection limit); second, to avoid introducing a large error in extrapolating to the epochs of our observations;

- temporal index $\alpha$ is available to extrapolate to the first epoch of our observations of GRB 040223 assuming that the light curve can be modelled by $F(t, v) \propto t^{-\alpha} v^{-\beta}$; in case of a temporal break, only the index valid for our epochs of observations is retained;

- the $R$ or $K_{\mathrm{s}}$ magnitude is given, with the delay preferably close to our epochs of observation; the spectral index $\beta$, if given, is used to extrapolate from $R$ to $K_{\mathrm{s}}$ when necessary for comparisons with the $K_{\mathrm{s}}$ band, and vice-versa.

Adopting the spectral index, we extrapolate the magnitudes to the $K_{\mathrm{S}}$ band and correct for absorption in the Galaxy (yielding $A_{R}>1$ for GRB 991216, GRB 011121, GRB 030528, GRB 031203 and GRB 041219A), using the $E(B-V)$ values from the FIRAS maps, corrected according to Dutra et al. (2003). Using the temporal index, we then extrapolate these computed magnitudes to the epochs of observation of GRB 040223 and GRB 040624. The temporal curve may deviate from a power-law behaviour (e.g. Tagliaferri et al. 2005a): however in the present case we compare magnitudes far from the early times. Galactic extinction is subject to inaccuracy, especially at low Galactic latitudes. However, the afterglow of GRB 011121 is the only case for which we made an extrapolation to the $K$ band with $A_{R}$ (Galaxy) $>1$. The lowest value is that of GRB 021211, which is $A_{R}$ (Galaxy) = 0.067. Estimating the extinction within the host galaxy for the afterglows of Table 4 is beyond the scope of this paper. However, the indications are that the reddening might be low. In fact in the rest frame of the host, $A_{\mathrm{V}}$ (host) = $0.21 \pm 0.12$ for GRB 971214 and consistent with zero for GRB 980519, GRB 990123, and GRB 990510 (Stratta et al. 2004). For GRB 020813, Savaglio \& Fall (2004) infer a 
Table 4. A compilation of afterglow data for 39 GRBs. References are given in superscripts. Delay times close to our epochs of observation for GRB 040223 and GRB 040624 are preferred.

\begin{tabular}{|c|c|c|c|c|c|c|}
\hline Id & Temporal index & Spectral index & $R$ & $\Delta T_{R}(\mathrm{~h})$ & $K$ & $\Delta T_{K}(\mathrm{~h})$ \\
\hline GRB 970228 & $1.1 \pm 0.1^{(1)}$ & $0.61 \pm 0.32$ & $20.83(2)$ & 16.88 & & \\
\hline GRB 971214 & $1.20 \pm 0.02^{(3)}$ & $0.93 \pm 0.060^{(4)}$ & $22.06^{(3)}$ & 12.89 & $18.03^{(5)}$ & 3.5 \\
\hline GRB 980326 & $2.0 \pm 0.1^{(5)}$ & $0.8 \pm 0.4^{(5)}$ & $21.25^{(5)}$ & 11.1 & & \\
\hline GRB 980329 & $1.21 \pm 0.13^{(6)}$ & & $23.6^{(6)}$ & 19.92 & & \\
\hline GRB 980519 & $2.3 \pm 0.12^{(7)}$ & $1.4 \pm 0.3^{(7)}$ & $20.28^{(7)}$ & 15.59 & & \\
\hline GRB 990123 & $1.1 \pm 0.03^{(8)}$ & $0.8 \pm 0.1^{(8)}$ & $20.0^{(8)}$ & 13.2 & $18.29^{(8)}$ & 29.47 \\
\hline GRB 990308 & $1.2 \pm 0.1^{(9)}$ & & $18.14^{(9)}$ & 3.34 & & \\
\hline GRB 990510 & $0.76 \pm 0.01^{(10)}$ & $0.61 \pm 0.12^{(10)}$ & $18.9^{(11)}$ & 14.99 & & \\
\hline GRB 990712 & $0.97 \pm 0.05^{(12)}$ & $0.7 \pm 0.1(12)$ & $20.175(12)$ & 10.38 & & \\
\hline GRB 991216 & $1.36 \pm 0.04^{(13)}$ & $0.58 \pm 0.08^{(13)}$ & $19.56^{(13)}$ & 22.5 & $15.01^{(13)}$ & 13.16 \\
\hline GRB 000926 & $1.69 \pm 0.2^{(14)}$ & $1.42 \pm 0.06^{(14)}$ & $19.326^{(14)}$ & 20.7 & & \\
\hline GRB 010222 & $0.8 \pm 0.05^{(15)}$ & $1.3 \pm 0.05^{(15)}$ & $18.67^{(15)}$ & 5.85 & & \\
\hline GRB 010921 & $1.59 \pm 0.18^{(16)}$ & $2.22 \pm 0.23^{(16)}$ & $19.4^{(17)}$ & 21.8 & & \\
\hline GRB 011121 & $1.72 \pm 0.05^{(18)}$ & $0.66 \pm 0.13^{(18)}$ & $19.06^{(18)}$ & 10.37 & & \\
\hline GRB 011211 & $0.83 \pm 0.04^{(19)}$ & $0.61 \pm 0.15^{(19)}$ & $20.51^{(19)}$ & 13.01 & & \\
\hline GRB 020124 & $1.6 \pm 0.04^{(20)}$ & $1.43 \pm 0.14^{(20)}$ & $18.376^{(20)}$ & 1.88 & & \\
\hline GRB 020405 & $1.54 \pm 0.06^{(21)}$ & $1.3 \pm 0.2^{(21)}$ & $20.17^{(21)}$ & 23.62 & & \\
\hline GRB 020813 & $0.76 \pm 0.05^{(22)}$ & $1.04 \pm 0.03^{(22)}$ & $18.49^{(22)}$ & 3.97 & & \\
\hline GRB 021211 & $1.11 \pm 0.01^{(23)}$ & $0.6 \pm 0.2^{(23)}$ & $22.2^{(23)}$ & 11.05 & & \\
\hline GRB 030226 & $0.50 \pm 0.35^{(24)}$ & $0.7 \pm 0.03^{(24)}$ & $19.9^{(24)}$ & 15.46 & $16.69^{(24)}$ & 8.51 \\
\hline GRB 030328 & $1.2 \pm 0.1^{(25)}$ & & $20.91(25)$ & 15.63 & & \\
\hline GRB 030329 & $0.89 \pm 0.01^{(26)}$ & $0.71^{(27)}$ & $15.02^{(27)}$ & 9.08 & & \\
\hline GRB 030429 & $0.95 \pm 0.03^{(28)}$ & $0.36 \pm 0.12^{(28)}$ & $20.86^{(28)}$ & 13.15 & $17.7^{(28)}$ & 13.63 \\
\hline GRB 030528 & $1.2^{(29)}$ & & $18.7^{(30)}$ & 2.38 & $18.6^{(29)}$ & 16.6 \\
\hline GRB 031203 & $2.0^{(31)}$ & $2.36 \pm 0.02^{(31)}$ & & & $17.56^{(31)}$ & 9.0 \\
\hline GRB 040924 & $0.7^{(32)}$ & & $18.67^{(33)}$ & 6.7 & $17.5^{(34)}$ & 2.4 \\
\hline GRB 041006 & $1.32 \pm 0.02^{(35)}$ & $0.45^{(36)}$ & $20.931^{(37)}$ & 14.94 & & \\
\hline GRB 041219A & $0.8^{(38)}$ & & & & $16.5^{(39)}$ & 24.24 \\
\hline GRB 041223 & $1.1 \pm 0.1^{(40)}$ & $0.6 \pm 0.1^{(40)}$ & $20.81^{(41)}$ & 14.4 & & \\
\hline GRB 050124 & $1.45 \pm 0.25^{(40)}$ & $0.4 \pm 0.2^{(40)}$ & & & $19.66^{(41)}$ & 24.5 \\
\hline GRB 050315 & $0.57^{(42)}$ & & $20.9^{(41)}$ & 11.6 & & \\
\hline GRB 050319 & $0.6^{(43)}$ & & $20.14^{(41)}$ & 8.7 & & \\
\hline GRB 050401 & $1^{(44)}$ & & $23.18^{(44)}$ & 11.39 & & \\
\hline GRB 050408 & $0.68 \pm 0.06^{(45)}$ & & $21.6^{(45)}$ & 8.75 & & \\
\hline GRB 050502A & $1.44 \pm 0.2^{(46)}$ & & $19.1^{(47)}$ & 5.83 & & \\
\hline GRB 050505 & $0.7^{(48)}$ & & $21.5^{(49)}$ & 7.83 & $18.1^{(50)}$ & 6.82 \\
\hline GRB 050525A & $1.38^{(51)}$ & & $18.1^{(52)}$ & 5.2 & $16.15^{(52)}$ & 5.2 \\
\hline GRB 050603 & $1.97^{(53)}$ & & $16.5^{(54)}$ & 3.4 & & \\
\hline GRB 050607 & $0.5^{(55)}$ & $1.5^{(55)}$ & $22.7^{(56)}$ & 1.27 & & \\
\hline
\end{tabular}

(1) Fruchter et al. (1999); (2) Guarnieri et al. (1997); ${ }^{(3)}$ Diercks et al. (1998); ${ }^{(4)}$ Reichart (1998); (5) Gorosabel et al. (1998), (5) Bloom et al. (1999); ${ }^{(6)}$ Reichart et al. (1999); ${ }^{(7)}$ Vrba et al. (2000); ${ }^{(8)}$ Kulkarni et al. (1999); ${ }^{(9)}$ Schaefer et al. (1999); ${ }^{(10)}$ Stanek et al. (1999); ${ }^{(11)}$ Harrison et al. (1999); ${ }^{(12)}$ Sahu et al. (2000); ${ }^{(13)}$ Garnavich et al. (2000); ${ }^{(14)}$ Fynbo et al. (2001b); ${ }^{(15)}$ Stanek et al. (2001); ${ }^{(16)}$ Price et al. (2002); ${ }^{(17)}$ Park et al. (2001); ${ }^{(18)}$ Garnavich et al. (2003); ${ }^{(19)}$ Holland et al. (2002); ${ }^{(20)}$ Berger et al. (2002); ${ }^{(21)}$ Masetti et al. (2003); (22) Covino et al. (2003); (23) Pandey et al. (2003); ${ }^{(24)}$ Klose et al. (2004); ${ }^{(25)}$ Andersen et al. (2003); (26) Torii et al. (2003); (27) Matheson et al. (2003); (28) Jakobsson et al. (2004); (29) Rau et al. (2004); ${ }^{(30)}$ Ayani \& Yamaoka (2003); ${ }^{(31)}$ Malesani et al. (2004); ${ }^{(32)}$ Fox (2004); (33) Hu et al. (2004); (34) Terada \& Akiyama (2004); ${ }^{(35)}$ Price et al. (2004); ${ }^{(36)}$ computed from Da Costa \& Noel (2004); ${ }^{(37)}$ Stanek et al. (2005); (38) Moon et al. (2004a); ${ }^{(39)}$ Moon et al. (2004b); ${ }^{(40)}$ Berger et al. (2005a); ${ }^{(41)}$ Berger et al. $(2005 b) ; ~{ }^{(42)}$ Cobb \& Bailyn (2005a); ${ }^{(43)}$ Torii (2005); ${ }^{(44)}$ D'Avanzo et al. (2005); ${ }^{(45)}$ Kosugi et al. (2005); ${ }^{(46)}$ Mirabal et al. (2005a); ${ }^{(47)}$ Fox (2005); ${ }^{(48)}$ Klotz et al. (2005); ${ }^{(49)}$ Chapman et al. (2005); (50) Rol et al. (2005a); ${ }^{(51)}$ Mirabal et al. (2005b); ${ }^{(52)}$ Cobb \& Bailyn (2005b); ${ }^{(53)}$ Brown et al. (2005); ${ }^{(54)}$ Berger \& McWilliams (2005); ${ }^{(55)}$ Rhoads (2005); (56) Halpern et al. (2005).

relatively high extinction of $A_{\mathrm{V}}$ (host) $\simeq 0.4$, but with weak dependence on wavelength, and therefore little impact on the power-law shape of the spectrum. This suggests that the extinction within the host is small, at least for the afterglows listed in Table 4 and observed in the $R$ band.
The results are presented in Figs. 10 and 11 in $R$ and $K_{\mathrm{s}}$ bands. For each epoch we show the observed magnitude limit. For GRB 040223 we report both the absorbed and unabsorbed value (the absorption for GRB 040624 can be neglected here). 


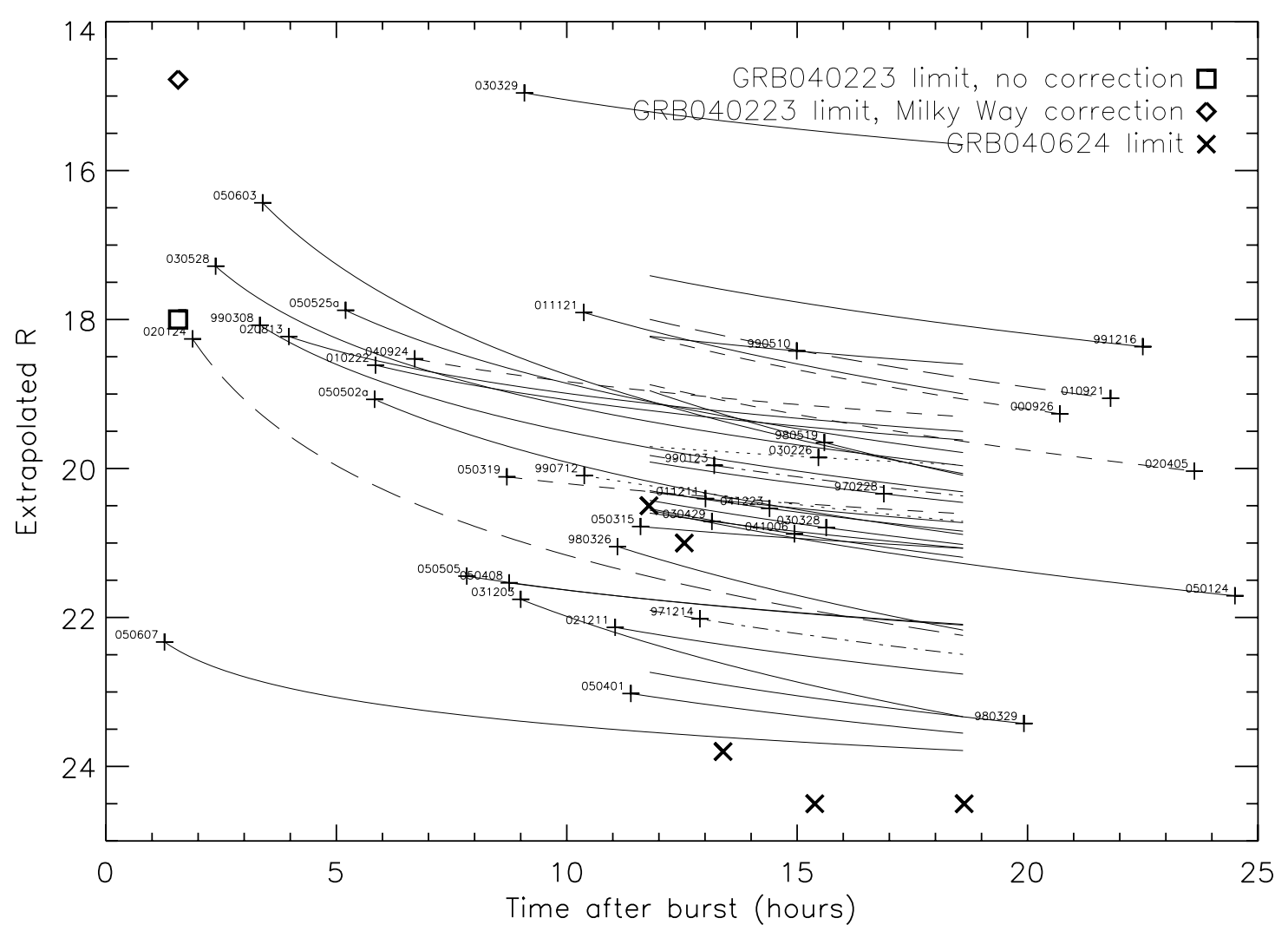

Fig. 10. Light curves of the afterglows listed in Table 4 with magnitudes extrapolated to the $R$ band (when necessary) after correction for Galactic absorption. The cross is placed at the time of observation. For clarity, different line styles are used to indicate the temporal power-law decay extrapolations, which only cover our observation epochs. The diamond and the square indicate the magnitude limits for GRB 040223 with and without correction for Galactic extinction, respectively, at 2- $\sigma$ (Gomboc et al. 2004). The crosses indicate the magnitude limits for GRB 040624. The first two come from the GCNs (Piccioni et al. 2004; Gorosabel et al. 2004). The last three points are our 3- $\sigma$ limits.

For GRB 040624, our deep 3- $\sigma$ limits reported in Fig. 10 indicate that we are facing a faint afterglow, even fainter than the Swift afterglow of GRB 050607. Indeed, Gaussian fits of the distribution of the extrapolated magnitudes shown in Fig. 10 at our observation epochs indicate that the afterglow of GRB 040624 is fainter by at least $2.7 \sigma$. As the sample of bursts in Fig. 10 is constructed to limit the bias towards bright afterglows, this indicates that the afterglow of GRB 040624 is fainter than normal. This is all the more interesting because this burst was very well placed for an observational campaign: near the celestial equator and at high Galactic latitude. We can then consider GRB 040624 as a very good example of a dark burst. Given the lack of observations at earlier epochs and at other wavelengths, the causes of its darkness cannot be properly ascertained.

For GRB 040223, our observations in the $r$ band are not so deep due to the location of the GRB towards the Galactic plane and the fairly long delay time for the $K_{\mathrm{s}}$ measurements. However, our limits (and especially the first epoch one, which is the more stringent) indicate that the burst is faint in the $K_{\mathrm{s}}$ band, as only a few GRBs have extrapolated magnitudes similar to our limit (i.e. GRB 971214, GRB 021211 and GRB 050607). In the $J$ and $H$ band, because of the Galactic absorption, our magnitude limits are less restrictive. Note that our group has already detected the faint afterglow of GRB 040422 with the VLT/ISAAC (Filliatre et al. 2005), with an observed magnitude $K_{\mathrm{s}}=18.0 \pm 0.1$ and a Galactic absorption of $A_{K}=0.5$ similar to that for GRB 040223. However, that observation was much quicker, $1.9 \mathrm{~h}$ after the burst. Assuming a temporal decay index of 1, the afterglow of GRB 040422 would have been $K_{\mathrm{s}}=20.3$ at our first epoch of observation of GRB 040223 and much fainter than our detection limit. The case of GRB 051001, with a $3 \sigma$ magnitude limit of $K_{\mathrm{s}}=20.3$ only $1.40 \mathrm{~h}$ after the trigger, (Rol et al. 2005b) is even more extreme, and demonstrates that observing within the first few hours is mandatory in the quest for faint afterglows.

\subsection{GRB 040223: from the X-ray to the NIR afterglow}

The power-law indices of the X-ray afterglow of GRB 040223 are $\alpha_{\mathrm{X}}=0.9 \pm 0.1$ and $\beta_{\mathrm{X}}=1.60 \pm 0.15$ and are not compatible with the closure relation in the form $\alpha+b \beta+c=$ 0 for isotropic expansion into a homogenous medium (Sari et al. 1998), isotropic expansion into a wind-stratified medium (Chevalier \& Li 1999) or a collimated expansion into a homogenous or wind-stratified medium (Sari et al. 1999), the cooling frequency being bluewards or redwards the X-ray domain. This fact can be seen in Fig. 2 of Piro (2005), with the other, less compelling, case of GRB 980703.

The extremely high hydrogen column density derived from the spectrum of the X-ray afterglow is significantly higher than 


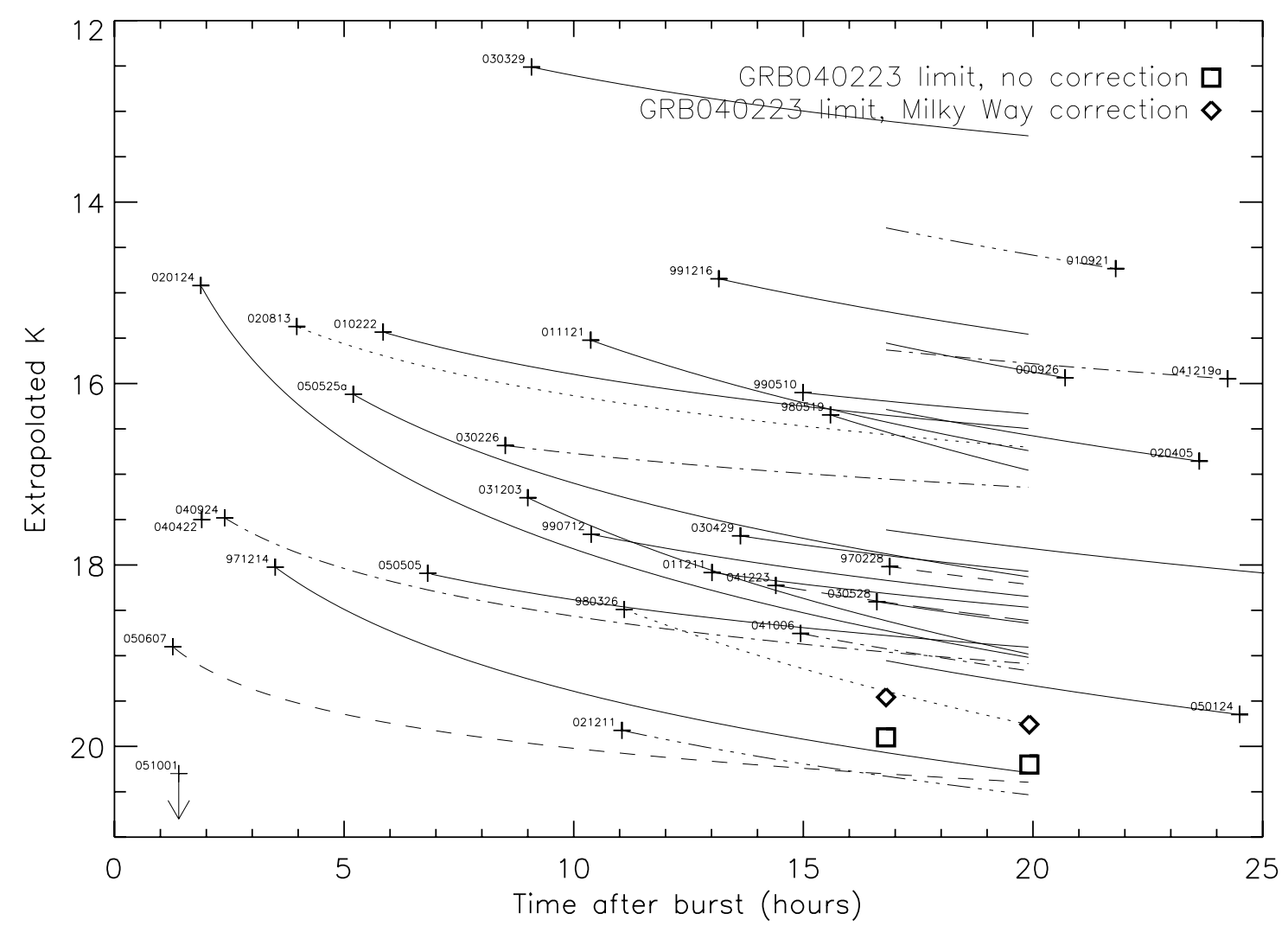

Fig. 11. Light curves of the afterglows listed in Table 4 with magnitudes extrapolated to the $K_{\mathrm{s}}$ band (when necessary) after correction for Galactic absorption. The cross is placed at the time of observation. For clarity, different line styles are used to indicate the temporal powerlaw decay extrapolations, which only cover our observation epochs. The diamonds and the squares indicate our 3- $\sigma$ magnitude limits with and without correction from Galactic extinction, respectively. The $K_{\mathrm{s}}$ magnitude for GRB 040422 (Filliatre et al. 2005) and the limit for GRB 051001 (Rol et al. 2005b) are also reported.

the estimated Galactic hydrogen column density. Assuming for simplicity that there is no significant absorption (in X-rays, optical and NIR) between our Galaxy and the GRB host, this strongly suggests that intrinsic absorption, in the restframe of the GRB, is present at a significant level. The correction needed in the optical/NIR bands to take this absorption into account, depends mainly on two unknown factors. The first one is the redshift of the GRB, which is required to relate the observed wavelength to the absorbed emitted wavelength, and also to estimate properly the hydrogen column density in the host galaxy. It is possible to use the spectrum of the X-ray afterglow to put constraints on the redshift of the burst and the intrinsic column density. For that, a redshifted model for the interstellar absorption, including a series of photoelectric absorption edges is fitted to the X-ray spectrum assuming solar system abundances. The resulting best fit value for the redshift is $z \sim 0.35$, for a corresponding intrinsic column density $N_{\mathrm{H}}^{\text {host }}(z) \sim 2 \times 10^{22} \mathrm{~cm}^{-2}$ (in addition to the previously quoted value $\left.N_{\mathrm{H}}^{\text {Galaxy }}=(6.6 \pm 2) \times 10^{21} \mathrm{~cm}^{-2}\right)$, but with no significant improvement in the quality of the fit with respect to the simple absorbed power-law model $\left(\chi_{v}^{2}=0.93,113\right.$ d.o.f. $)$. As the photoelectric cross-section scales roughly as $E^{\sim-2.6}$, we have, to first approximation $N_{\mathrm{H}}^{\text {host }}(z)=N_{\mathrm{H}}^{\text {host }}(z=0)(1+z)^{2.6}$, where $N_{\mathrm{H}}^{\text {host }}(z=0)=N_{\mathrm{H}}^{\text {measured }}-N_{\mathrm{H}}^{\text {Galaxy }}$ (Stratta et al. 2004). Indeed, the redshift and the column density parameters in the fit are degenerate, as may be seen from the contour plot of Fig. 12. In any case, the data allows us to estimate that the gas column is at least $N_{\mathrm{H}}^{\text {host }}(z=0)=10^{22} \mathrm{~cm}^{-2}$, while the redshift is no larger than $1.7\left(N_{\mathrm{H}}^{\text {host }}(z)<1.1 \times 10^{23} \mathrm{~cm}^{-2}\right)$, at $90 \%$ confidence level for 2 parameters of interest. For $z>1.7$ the fit turns out to be unacceptable since we try to fit the Fe edge in the portion of the spectrum which has larger statistics (below $2.5 \mathrm{keV}$ ) and which does not exhibit an absorption edge. Note however that the fit is good again for $z>7$, for which there is no absorption edge in the $1-4 \mathrm{keV}$ range. Hence, the $\mathrm{X}$-ray data constrain the redshift to be $0<z<1.7$, or $z>7$, assuming solar abundances.

The second factor is the relationship between the hydrogen column density and the absorption law that is valid in the optical/NIR domain. We will consider two hypotheses: first, the host galaxy behaves like our Galaxy, and the relationships of Predehl \& Schmitt (1995) and Cardelli et al. (1989) with $R_{\mathrm{V}}=3.1$ still hold; second, the host galaxy behaves as the Small Magellanic Clouds, with $N_{\mathrm{H}} / A_{\mathrm{V}}=1.6 \times 10^{22} \mathrm{~cm}^{-2}$ (Weingartner \& Draine 2001) and use the extinction law fitted by Pei (1992) with $R_{\mathrm{V}}=2.93$. This hypothesis is a reasonable choice for the host, as far as absorption properties are concerned (Stratta et al. 2004).

With these two hypotheses, and assuming that $N_{\mathrm{H}}^{\text {host }}(z)=$ $N_{\mathrm{H}}^{\text {host }}(z=0)(1+z)^{2.6}$, we extrapolated the luminosity of the $\mathrm{X}$-ray afterglow to the $r$ to $K$ bands, and compared the result 
Table 5. The magnitudes of the optical/NIR afterglow of GRB 040223 predicted by extrapolation of the X-ray afterglow, assuming that the host is similar to the Galaxy. The observed limits are reported for comparison.

\begin{tabular}{llcccccc}
\hline \hline & $r$ & $J, 1$ st epoch & $J, 2$ nd epoch & $H, 1$ st epoch & $H, 2$ nd epoch & $K_{\mathrm{s}}, 1$ st epoch & $K_{\mathrm{s}}, 2$ nd epoch \\
\hline $\begin{array}{l}\text { Observed limits } \\
\text { Simple extrapolation }\end{array}$ & 18 & 20.9 & 21.1 & 19.8 & 20.4 & 19.9 & 20.2 \\
$z=0$ & $19.5 \pm 2$ & $16.0 \pm 2$ & $16.2 \pm 2$ & $14.1 \pm 2$ & $14.3 \pm 2$ & $12.5 \pm 2$ & $12.6 \pm 2$ \\
$z=0.9$ & $58 \pm 11$ & $37 \pm 6$ & $37 \pm 6$ & $27 \pm 4$ & $27 \pm 4$ & $20.7 \pm 3$ & $20.9 \pm 3$ \\
\hline Cooling break, fast cooling & & & & & & \\
$z=0, v_{\mathrm{c}}=7.5 \times 10^{16} \mathrm{~Hz}$ & $25.6 \pm 1$ & $22.8 \pm 1$ & $23.0 \pm 1$ & $21.2 \pm 1$ & $21.4 \pm 1$ & $20.0 \pm 1$ & $20.1 \pm 1$ \\
$z=0, v_{\mathrm{c}}=1.2 \times 10^{17} \mathrm{~Hz}$ & $26.2 \pm 1$ & $23.4 \pm 0.5$ & $23.6 \pm 0.5$ & $21.9 \pm 0.4$ & $22.0 \pm 0.4$ & $20.6 \pm 0.3$ & $20.7 \pm 0.3$ \\
\hline Cooling break, slow cooling & & & & & & \\
$z=0, v_{\mathrm{c}}=1.2 \times 10^{17} \mathrm{~Hz}$ & $22.5 \pm 2$ & $19.3 \pm 2$ & $19.5 \pm 2$ & $17.6 \pm 2$ & $17.8 \pm 2$ & $16.1 \pm 2$ & $16.3 \pm 2$ \\
$z=0.6, v_{\mathrm{c}}=1.2 \times 10^{17} \mathrm{~Hz}$ & $42 \pm 6$ & $29 \pm 3$ & $29 \pm 3$ & $23.6 \pm 2$ & $23.7 \pm 2$ & $19.9 \pm 2$ & $20.1 \pm 2$ \\
\hline
\end{tabular}

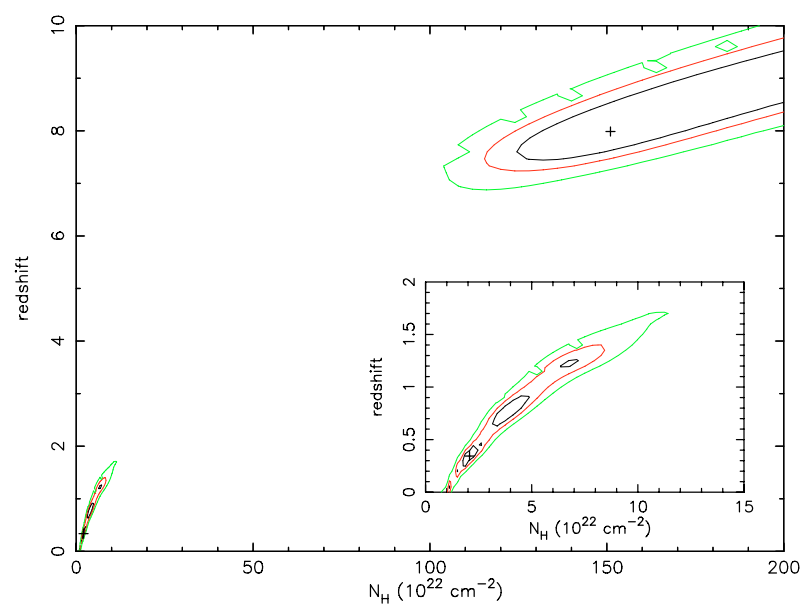

Fig. 12. For GRB 040223, confidence contours (at 68\%, $90 \%$ and $99 \%$ level for two parameters of interest) for the redshift $z$ and the column density $N_{\mathrm{H}, z}$ of the redshifted absorber, derived from the fit to the EPIC spectra. The inner plot is an enlargement of the low- $z$ region.

with our observational limits. Assuming a synchrotron emission for the afterglow, we considered two cases:

1. both the photon and time indices derived in the X-ray are valid in the $r$ to $K$ bands, at the epochs of observations; the degree of freedom here is the redshift;

2. the cooling break frequency happens to lie between the $\mathrm{X}$-ray domain and the optical/NIR domain at the observation epochs in the optical/NIR, with two different spectral distributions (Sari et al. 1998):

(a) the fast cooling case, for which $\beta_{\text {opt, NIR }}=0.5$;

(b) the slow cooling case, for which $\beta_{\mathrm{opt}, \mathrm{NIR}}=\beta_{\mathrm{X}}-0.5$.

The extrapolated magnitudes are listed in Tables 5 and 6, where the error bars are computed by simulations taking into account the uncertainties in the parameters (the X-ray flux, $\beta_{\mathrm{X}}$, $\alpha_{\mathrm{X}}, N_{\mathrm{H}}^{\text {measured }}$ and $N_{\mathrm{H}}^{\text {Galaxy }}$ ). In all cases, the most stringent constraints are provided by the observations in the $K_{\mathrm{s}}$ band. We can also assume that a temporal break occurred between the epoch of the X-ray observations, and the epoch of the $J, H, K_{\mathrm{s}}$ observations. This case can combine with the previous ones, independently of the modelling of the absorption in the host galaxy and of the redshift. However, even assuming that the temporal index steepened to $\alpha=2$ just at the end of the X-ray observations, the extrapolated NIR afterglow would be brighter by less than $1 \mathrm{mag}$, which is smaller than the error bars reported in Tables 5 and 6 . We therefore do not consider further the possibility of a temporal break.

These results are compatible with the hypothesis that the synchrotron model is valid and that the X-ray and optical/NIR afterglow come from the same component, although the hypothesis that the afterglows are due to two different components cannot be excluded either. Indeed, the extrapolations are compatible with the observational limits in the following cases:

1. $z \geqq 0.9$ if the host is similar to the Galaxy, $z \geqq 2.1$ if the host is similar to the SMC;

2. with a cooling break,

(a) with a fast cooling, for a cooling break frequency $v_{\mathrm{c}}>$ $7.5 \times 10^{16} \mathrm{~Hz}\left(\lambda_{\mathrm{c}}<0.01 \mu \mathrm{m}\right)$ if the host is similar to the Galaxy; for a cooling break frequency at the lower edge of the X-ray band $\left(E_{\mathrm{c}}=0.5 \mathrm{keV}\right.$, i.e. $v_{\mathrm{c}}=1.2 \times$ $10^{17} \mathrm{~Hz}$ ), or for $v_{\mathrm{c}}>7.5 \times 10^{16} \mathrm{~Hz}$ for $z \geqq 0.8$ if the host is similar to the SMC;

(b) with a slow cooling, for $v_{\mathrm{c}}=1.2 \times 10^{17} \mathrm{~Hz}$ and $z \geqq 0.6$ if the host is similar to the Galaxy, $z \geqq 1.8$ if the host is similar to the SMC.

However the possibility that these two GRBs are intrinsically sub-luminous with faint afterglows cannot be excluded. Redshifts above 0.9 are common in GRBs and case 1, because it does not involve fine tuning between the epochs of observations and a break in the spectrum, is the simplest explanation. In conclusion, our magnitude limits are compatible with a simple extrapolation of the properties of the X-ray afterglow for an acceptable redshift range, independent of the host galaxy.

\section{Conclusions}

Our main results on GRB 040223 and GRB 040624 are as follows:

- These two bursts are among the weakest and longest detected by INTEGRAL. The $\gamma$-ray spectra are well fit by power-laws with steep spectral indices suggesting that both are X-ray rich GRBs. 
Table 6. The magnitudes of the optical/NIR afterglow of GRB 040223 predicted by extrapolation of the X-ray afterglow, assuming that the host is similar to the SMC. The observed limits are reported for comparison.

\begin{tabular}{llcccccc}
\hline \hline & $r$ & $J, 1$ st epoch & $J, 2$ nd epoch & $H, 1$ st epoch & $H, 2$ nd epoch & $K_{\mathrm{s}}, 1$ st epoch & $K_{\mathrm{s}}, 2$ nd epoch \\
\hline Observed limits & 18 & 20.9 & 21.1 & 19.8 & 20.4 & 19.9 & 20.2 \\
Simple extrapolation & & & & & & \\
$z=0$ & $15.8 \pm 2$ & $14.6 \pm 2$ & $14.7 \pm 2$ & $13.2 \pm 2$ & $13.3 \pm 2$ & $11.9 \pm 2$ & $12.0 \pm 2$ \\
$z=2.1$ & $50 \pm 9$ & $32 \pm 5$ & $33 \pm 5$ & $26 \pm 4$ & $26 \pm 4$ & $20.4 \pm 3$ & $20.6 \pm 3$ \\
\hline Cooling break, fast cooling & & & & & & \\
$z=0.8, v_{\mathrm{c}}=7.5 \times 10^{16} \mathrm{~Hz}$ & $25.6 \pm 1$ & $23.2 \pm 0.6$ & $23.3 \pm 0.6$ & $21.5 \pm 0.4$ & $21.6 \pm 0.4$ & $20.1 \pm 0.4$ & $20.2 \pm 0.4$ \\
$z=0, v_{\mathrm{c}}=1.2 \times 10^{17} \mathrm{~Hz}$ & $22.5 \pm 0.3$ & $22.0 \pm 0.3$ & $22.2 \pm 0.3$ & $20.9 \pm 0.3$ & $21.1 \pm 0.3$ & $20.0 \pm 0.3$ & $20.2 \pm 0.3$ \\
\hline Cooling break, slow cooling & & & & & & \\
$z=0, v_{\mathrm{c}}=1.2 \times 10^{17} \mathrm{~Hz}$ & $18.8 \pm 1$ & $17.9 \pm 1$ & $18.1 \pm 1$ & $16.7 \pm 1$ & $16.8 \pm 1$ & $15.5 \pm 1$ & $15.7 \pm 1$ \\
$z=1.8, v_{\mathrm{c}}=1.2 \times 10^{17} \mathrm{~Hz}$ & $39 \pm 6$ & $28.6 \pm 3$ & $28.7 \pm 3$ & $24.1 \pm 2$ & $24.3 \pm 2$ & $20.3 \pm 2$ & $20.5 \pm 2$ \\
\hline
\end{tabular}

- For GRB 040223, an X-ray afterglow was detected by XMM-Newton. The spectral properties indicate an extremely high column density of $(1.60 \pm 0.15) \times 10^{22} \mathrm{~cm}^{-2}$, much higher than the expected contribution from the Galaxy. This result suggests significant absorption in the host galaxy of the burst.

- The magnitude limits we obtained in the NIR for GRB 040223 are compatible with a simple extrapolation of the properties of the X-ray afterglow.

- For GRB 040624, we obtained magnitude limits in the optical that are fainter than the very faint end of the distribution of the magnitudes of a compilation of 39 promptly observed counterparts.

In conclusion these two GRBs can be classified as bursts with a dark afterglow. For GRB 040223, the cause of darkness seems to be the intrinsic absorption. We remark that a very high dust extinction $\left(A_{\mathrm{V}} \sim 30-50\right)$ has also been proposed as a possible cause of the discrepancy (a factor 3-10) between the observed NIR rate of core-collapse supernovae and the rate estimated from the far-IR luminosity of the parent galaxies (Mannucci et al. 2003; Maiolino et al. 2002). Given the relationships between long GRBs and core-collapse supernovae (e.g. Della Valle 2005), we may be led to the conclusion that a similar effect is at play also in this case and that most "dark" bursts are in fact highly extincted "normal" GRBs.

The increasing use of large diameter telescopes, with instruments operating in the infrared, involved in a comprehensive observational programme with reduced delay may increase the number of similar bursts. The magnitude of GRB 040422 (Filliatre et al. 2005) and more dramatically the limit on GRB 051001 (Rol et al. 2005b) stress the clear fact that, when looking for faint afterglows, observing within the first few hours is mandatory.

Acknowledgements. We are very grateful to the ESO staff at Paranal for carefully performing all our observations, and for many useful suggestions. This research is supported by the Italian Space Agency (ASI), and is part of the GRB activity related to the Swift mission (contract ASI/I/R/390/02).

\section{References}

Andersen, M., Masi, G., Jensen, B. L., \& Hjorth, J. 2003, GCN 1992 Ayani, K., \& Yamaoka, M. 2003, GCN 2257

Barraud, C., Olive, J. F., \& Lestrade, J. P. 2003, A\&A, 400, 1021

Berger, E., \& McWilliams, A. 2005, GCN 3511

Berger, E., Kulkarni, S. R., Bloom, J. S., et al. 2002, ApJ, 581, 981

Berger, E., Fox, D. B., Kulkarni, S. R., et al. 2005a, ApJ, 629, 328

Berger, E., Kulkarni, S. R., Fox, D. B., et al. 2005b, ApJ, 634, 501

Bloom, J. S., Kulkarni, S. R., Djorgovski, S. G., et al. 1999, Nature, 401, 453

Breitfellner, M. G., Munuera, P. M., \& Martos, A. 2004, GCN 2530

Brown, P., Retter, A., Schady, P., et al. 2005, GCN 3549

Burenin, R. A., Vikhlinin, A. A., Terekov, O. V., et al. 1999, Ast. L., 25,411

Cardelli, J. A., Clayton, G. C., \& Mathis, J. S. 1989, ApJ, 345, 245

Chapman, R., Tanvir, N., Rol, E., et al. 2005, GCN 3375

Chevalier, R. A., \& Li, Z.-Y. 1999, ApJ, 520, 29

Chincarini, G., Zerbi, F., Antonelli, L. A., et al. 2003, The ESO Messenger, 113, 40

Chincarini, G., Moretti, A., Romano, P., et al. 2005

[arXiv:astro-ph/0506453]

Cobb, B. E., \& Bailyn, C. D. 2005a, GCN 3110

Cobb, B. E., \& Bailyn, C. D. 2005b, GCN 3506

Covino, S., Malesani, D., Tavecchio, F., et al. 2003, A\&A, 404, L5

Cusumano, G., Mangano, V., Chincarini, G., et al. 2005 [arXiv: astro-ph/0509737]

D’Avanzo, P., Fugazza, D., Melandri, A., et al. 2004, GCN 2632

D’Avanzo, P., Fugazza, D., Masetti, N., \& Pedani, M. 2005, GCN 3171

Da Costa, G., \& Noel, N. 2004, GCN 2789

De Luca, A., Mereghetti, S., Tiengo, A., \& Campana, S. 2004, GCN 2547

Della Valle, M. 2005, in Gamma-Ray Bursts in the Afterglow Era: 4th Workshop, held in Rome, September, 2004, Il Nuovo Cimento, in press [arXiv: astro-ph/0504517]

Devillard, N. 1997, the ESO Messenger, 87

Dickey, J., \& Lockman, F. J. 1990, ARA\&A, 28, 215

Diercks, A. H., Deutsch, E. W., Castander, F. J., et al. 1998, ApJ, 503, 105

Dutra, C. M., Ahumada, A. V., Clariá, J. J., et al. 2003, A\&A, 408, 287

Filliatre, P., D’Avanzo, P., Covino, S., et al. 2005, A\&A, 438, 793

Fiore, F., d'Elia, V., Lazzati, D., et al. 2005, ApJ, 624, 853

Fox, D. B. 2004, GCN 2741 
Fox, D. B. 2005, GCN 3442

Frontera, F., Amati, L., Costa, E., et al. 2000, ApJS, 127, 59

Fruchter, A., Pian, E., Thorsett, S., et al. 1999, ApJ, 516, 683

Fugazza, D., D’ Avanzo, P., Tagliaferri, G., et al. 2004, GCN 2617

Fynbo, J. U., Jensen, B. L., Gorosabel, J., et al. 2001a, A\&A, 369, 373

Fynbo, J. U., Gorosabel, J., Dall, T. M., et al. 2001b, A\&A, 373, 796

Garnavich, P. M., Jha, S., Pahre, M. A., et al. 2000, ApJ, 543, 61

Garnavich, P. M., Stanek, K. Z., Wyrzykowski, L., et al. 2003, ApJ, 582, 924

Giblin, T. W., van Paradijs, J., Kouveliotou, C., et al. 1999, ApJ, 524, 47

Gomboc, A., Marchant, J. M., Smith, R. J., Mottram, C. J., \& Fraser, S. N. 2004, GCN 2534

Gorosabel, J., Castro-Tirado, A. J., Willott, C. J., et al. 1998, A\&A, 335, L8

Gorosabel, J., Casanova, V., Verdes-Montenegro, L., et al. 2004, GCN 2615

Gotz, D., Mereghetti, D., Beck, M., Borkowski, J., et al. 2004, GCN 2525

Guarnieri, A., Bartolini, C., Masetti, N., et al. 1997, A\&A, 328, L13

Halpern, J. P., Kemp, J., \& Mirabal, N. 2005, GCN 3530

Harrison, F. A., Bloom, J. S., Frail, D. A., et al. 1999, ApJ, 523, 121

Holland, S. T., Soszynski, I., Gladders, M., et al. 2002, AJ, 124, 639

Hu, J. H., Lin, H. C., Huang, K., et al. 2004, GCN 2744

Hurley, K., Dingus, B. L., Mukherjee, R., et al. 1994, Nature, 372, 652

Israel, G. L., Covino, S., Tosti, G., et al. 2004, GCN 2531

Jakobsson, P., Hjorth, J., Fynbo, J. U., et al. 2004, A\&A, 427, 785

Klose, S., Greiner, J., Rau, A., et al. 2004, AJ, 128, 1942

Klotz, A., Boer, M., \& Atteia, J.-L. 2005, GCN 3403

Kosugi, G., Kawai, N., Aoki, K., et al. 2005, GCN 3263

Kulkarni, S. R., Djorgovski, S. G., Odewahn, S. C., et al. 1999, Nature, 398, 389

Lamb, D. Q. 2000, Phys. Rep., 333, 505

Lamb, D. Q., \& Reichart, D. E. 2000, ApJ, 536, 1

Lamb, D. Q., Ricker, J. R., Atteia, J.-L., et al. 2004, New Astron. Rev., 48,423

Lazzati, D., Covino, S., \& Ghisellini, G. 2002, MNRAS, 330, 583

Maiolino, R., Vanzi, L., Mannucci, F., et al. 2002, A\&A, 389, 84

Malesani, D., Tagliaferri, G., Chincarini, G., et al. 2004, ApJ, 609, 5

Mannucci, F., Maiolino, R., Cresci, G., et al. 2003, A\&A, 401, 519

Masetti, N., Palazzi, E., Pian, E., et al. 2003, A\&A, 404, 465

Matheson, T., Garnavich, P. M., Stanek, K. Z., et al. 2003, ApJ, 599, 394

McBreen, S., McBreen, B., Quilligan, F., \& Hanlon, L. 2002a, A\&A, 385, L19

McBreen, S., McBreen, B., Hanlon, L., \& Quilligan, F. 2002b, A\&A, 393, L29

McGlynn, S., McBreen, S., Hanlon, L., et al. 2005, in GammaRay Bursts in the Afterglow Era: 4th Workshop, held in Rome, September, 2004, Il Nuovo Cimento, in press [arXiv: astro-ph/0505349]

Mereghetti, S., \& Götz, D. 2005 [arXiv: astro-ph/0506198]

Mereghetti, S., Götz, D., Borkowski, J., Walter, R., \& Pedersen, H. 2003, A\&A, 411, L291

Mereghetti, S., Götz, D., Borkowski, J., et al. 2004a, in Proc. of the 5th INTEGRAL Workshop: The INTEGRAL Universe (Munich), ed. V. Schönfelder, G. Lichti, \& C. Winkler (ESA Special Publication), SP-552, 599

Mereghetti, S., Gotz, D., Beck, M., \& Borkowski, J. 2004b, GCN 2613 Mirabal, N., Boettcher, M., Shields, J., et al. 2005a, GCN 3363
Mirabal, N., Bonfield, D., \& Schawinski, K. 2005b, GCN 3488

Moon, D.-S., Cenko, S. B., \& Adams, J. 2004a, GCN 2876

Moon, D.-S., Cenko, S. B., \& Adams, J. 2004b, GCN 2884

Moran, L., Mereghetti, S., Götz, D., et al. 2005, A\&A, 432, 467

Nousek, J. A., Kouveliotou, C., Grupe, D., et al. 2005

[arXiv: astro-ph/0508332]

Pandey, S. B., Anupama, G. C., Sagar, R., et al. 2003, A\&A, 408, L21

Park, H. S., Williams, G., Barthelmy, S., et al. 2001, GCN 1131

Pei, Y. C. 1992, ApJ, 395, 130

Piccioni, A., Bartolini, C., Guarnieri, A., et al. 2004, GCN 2623

Piro, L. 2005, in Gamma-Ray Bursts in the Afterglow Era: 4th Workshop, held in Rome, September, 2004, Il Nuovo Cimento, in press [arXiv: astro-ph/0506020]

Predehl, P., \& Schmitt, J. H. M. M. 1995, A\&A, 293, 889

Price, P. A., Kulkarni, S. R., Berger, E., et al. 2002, ApJ, 571, 121

Price, P. A., Da Costa, G., \& Noel, N. 2004, GCN 2771

Quilligan, F., McBreen, B., Hanlon, L., et al. 2002, A\&A, 385, 377

Rau, A., Greiner, J., Klose, S., et al. 2004, A\&A, 427, 815

Reichart, D. E. 1998 [arXiv: astro-ph/9801139]

Reichart, D. E., Lamb, D. Q., Metzger, M. R., et al. 1999, ApJ, 517, 692

Reichart, D. E., \& Price, P. A. 2002, ApJ, 565, 174

Rhoads, J. 2005, GCN 3531

Rol, E., Tanvir, N., Levan, A., et al. 2005a, GCN 3372

Rol, N., Levan, A., Tanvir, E., et al. 2005b, GCN 4053

Sahu, K. C., Vreeswijk, P. M., Bakos, G., et al. 2000, ApJ, 540, 74

Sari, R., Piran, T., \& Narayan, R. 1998, ApJ, 497, 17

Sari, R., Piran, T., \& Halpern, J. P. 1999, ApJ, 519, 17

Savaglio, S., \& Fall, S. M. 2004, ApJ, 614, 293

Schaefer, B. E., Snyder, J. A., Hernandez, J., et al. 1999, ApJ, 524, 103

Schlegel, D., Finkbeiner, D., \& Davis, M. 1998, ApJ, 500, 525

Simoncelli, A., D' Avanzo, P., Campana, S., et al. 2004, GCN 2549

Soderberg, A. M., \& Frail, D. A. 2004, GCN 2532

Stanek, K. Z., Garnavich, P. M., Kaluzny, J., et al. 1999, ApJ, 522, 39

Stanek, K. Z., Garnavich, P. M., Jha, S., et al. 2001, ApJ, 563, 592

Stanek, K. Z., Garnavich, P. M., Nutzman, P. A., et al. 2005, ApJ, 626, 5

Stratta, G., Fiore, F., Antonelli, L. A., Piro, L., \& De Pasquale, M. 2004, ApJ, 608, 846

Strü der, L., Briel, U., Dennerl, K., et al. 2001, A\&A, 365, L18

Tagliaferri, G., Fugazza, D., Covino, S., et al. 2004, GCN 2535

Tagliaferri, G., Goad, M., Chincarini, G., et al. 2005a, Nature, 436, 985

Tagliaferri, G., Antonelli, L. A., Chincarini, G., et al. 2005b, A\&A, 443, L1

Terada, H., \& Akiyama, M. 2004, GCN 2742

Torii, K. 2005, GCN 3121

Torii, K., Kato, T., Yamaoka, H., et al. 2003, ApJ, 597, 101

Turner, M. J. L., Abbey, A., Arnand, N., et al. 2001, A\&A, 365, L27

Ubertini, P., Lebrun, F., Di Cocco, G., et al. 2003, A\&A, 411, L131

Vedrenne, G., Roques, J. P., Schönfelder, V., et al. 2003, A\&A, 411, L63

Vrba, F. J., Henden, A. A., Canzian, B., et al. 2000, ApJ, 528, 254

Weingartner, J. C., \& Draine, B. T. 2001, ApJ, 548, 296

Winkler, C., Courvoisier, T. J., Di Cocoo, G., et al. 2003, A\&A, 411, L1

Zerbi, F. M., Chincarini, G., Ghisellini, G., et al. 2001, Astron. Nachr., 322,275

Zhang, B., \& Mészáros, P. 2004, Int. J. Mod. Phys. A, 19, 2385 